\title{
Collagen type III glomerulopathy: A case report and review of 20 cases
}

\author{
JUNWU DONG $^{1 *}$, HONGLAN WEI $^{1 *}$, MIN HAN $^{2}$, YANG GUAN $^{3}$, YANG WU $^{1}$ and HUA LI ${ }^{1}$ \\ ${ }^{1}$ Department of Nephrology and Rheumatology, Puai Hospital; ${ }^{2}$ Department of Nephrology, Tongji Hospital; \\ ${ }^{3}$ Department of Pathology, Tongji Medical College, Huazhong University of Science and Technology, \\ Wuhan, Hubei 430000, P.R. China
}

Received March 1, 2014; Accepted September 12, 2014

DOI: $10.3892 /$ etm.2015.2695

\begin{abstract}
Collagen type III glomerulopathy is a non-immune-mediated glomerular disease, characterized by abnormal accumulation of type III collagen fibrils within the mesangial matrix and subendothelial space. The clinical manifestations of this disease are proteinuria, peripheral edema, hypertension and occasional progression to end-stage renal disease. Collagen type III glomerulopathy is extremely rare, and its etiology and pathogenesis remain elusive. To date, only case reports are available and the majority of these are from Japan. To investigate the idiographic features of collagen type III glomerulopathy in China, we report a case of collagen type III glomerulopathy with two differing renal biopsies and review 20 cases in China. The majority of the Chinese patients were adults. Thirty percent of the patients had nephrotic syndrome, and hypertension was observed in $75 \%$ of cases. Elevated creatinine was present in $45 \%$ of patients. The pathology of collagen type III glomerulopathy in the Chinese cases was similar to that observed in other ethnicities, although certain cases were IgA-positive by immunofluorescence microscopy, and electron-dense material could be observed in the mesangial area. The onset age, clinical manifestations and pathological features of the disease are not exactly the same in China as worldwide.
\end{abstract}

\section{Introduction}

In 1979, Arakawa et al first described a new glomerular disease with collagen fibrils in the mesangial matrix and

Correspondence to: Professor Junwu Dong, Department of Nephrology and Rheumatology, Puai Hospital, Tongji Medical College, Huazhong University of Science and Technology, 473 Hanzheng Street, Wuhan, Hubei 430000, P.R. China

E-mail: junwudongcn@126.com

*Contributed equally

Key words: collagen type III glomerulopathy, type III collagen fibril, proteinuria, China subendothelial space (1). Subsequently, in 1990, Ikeda et al proved that the collagen fibrils in the renal glomerulus were type III collagen fibrils by immunohistochemical staining (2). This new glomerular disease was officially named collagen type III glomerulopathy by Imbasciati et al in 1991 (3). The clinical manifestations of this disease are proteinuria, edema, hypertension and occasional progression to end-stage renal disease (4,5). The etiology and pathogenesis remain elusive. Although the majority of the initial studies were from Japan, there are currently studies from around the globe including Europe, South America, North America and Asia. However, studies from China are rare in the English literature, and the specific features of Chinese cases are yet to be summarized. Here, we report a case of collagen type III glomerulopathy with two differing renal biopsies and review 20 cases in China to investigate the idiographic features of collagen type III glomerulopathy in China. This study was conducted in accordance with the declaration of Helsinki, and with the approval of the Ethics Committee of Puai Hospital, Tongji Medical College, Huazhong University of Science and Technology (Wuhan, China). Written informed consent was obtained from the participant.

\section{Case report}

In July 2011, a 12-year-old male presented with a one-year history of edema and proteinuria. The patient was suffering lower extremity and periorbital edema associated with hypertension. He had no nail or patella dysplasia and no family history of renal disease. Laboratory results included urine protein levels of $2.7 \mathrm{~g} / 24 \mathrm{~h}(0.028-0.141 \mathrm{~g} / 24 \mathrm{~h})$ and no microscopic hematuria, hemoglobin levels of $86 \mathrm{~g} / 1$ (normal level 131-172 g/l), serum albumin levels of $26.3 \mathrm{~g} / 1$ (normal level 34-48 g/l), blood urea nitrogen levels of $7.92 \mathrm{mmol} / 1$ (normal level 2.9-8.2 mmol/1), creatinine levels of $122.8 \mu \mathrm{mol} / 1$ (normal level 62-115 $\mu \mathrm{mol} / 1$ ), elevated uric acid levels of $457.1 \mu \mathrm{mol} / 1$ (normal level 208-428 $\mu \mathrm{mol} / 1$ ), C3 levels of $0.61 \mathrm{~g} / 1$ (normal level 0.79-1.52 g/1) and normal C4 levels. Screening for auto-antibodies was negative. The first renal biopsy was performed on July 26, 2011, but only one extremely small specimen was taken as the patient was extremely anxious and the patient's blood pressure was elevated during the procedure. The specimen was processed by immunofluo- 
A

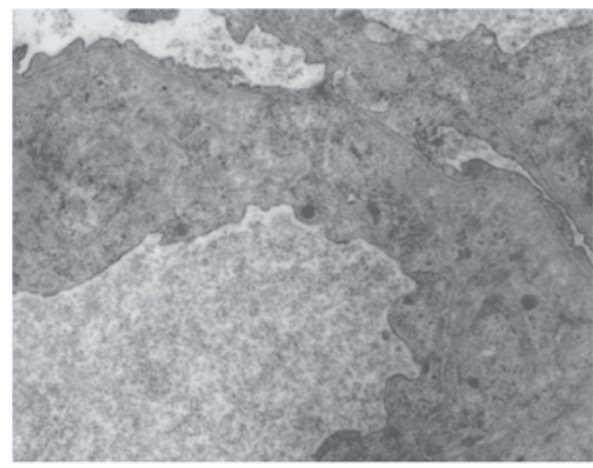

B

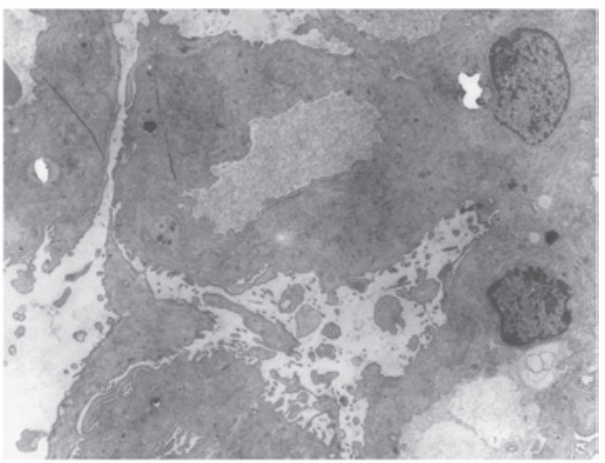

Figure 1. First renal biopsy. Electron microscopic images revealing no electron-dense or collagen fibrils deposists in the (A) subendothelial space (magnification, $\mathrm{x} 15,000)$ and (B) mesangial matrix (magnification, $\mathrm{x} 10,000$ ).
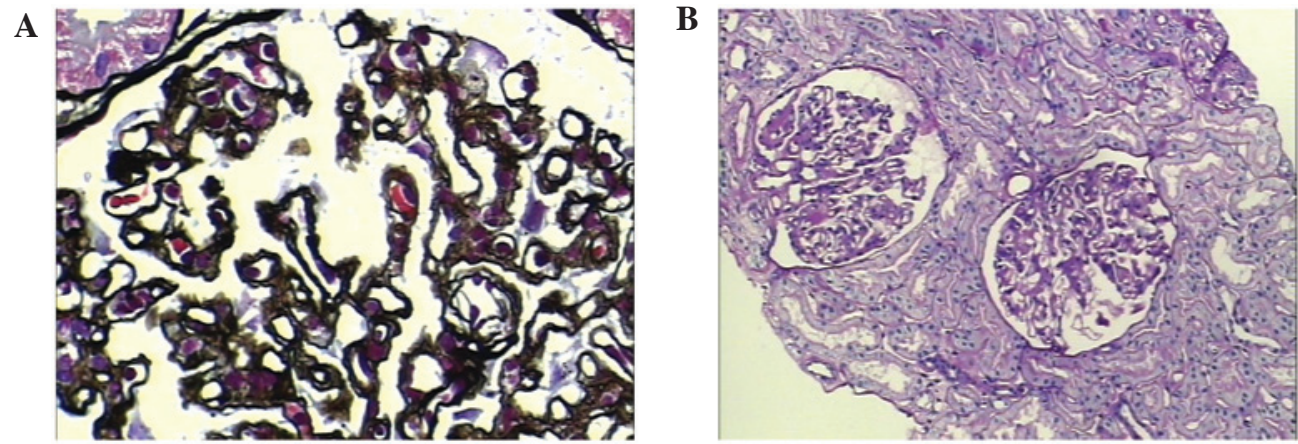

Figure 2. Second renal biopsy.(A) Light microscopy image revealing fake double-track in some segment of basal membrane (magnification, x400). (B) Light microscopy image revealing mild mesangial hypercellularity (magnification, $\mathrm{x} 200$ ).

A

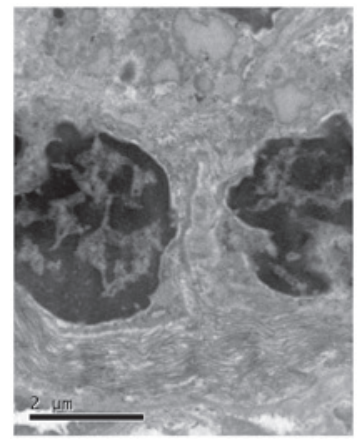

B

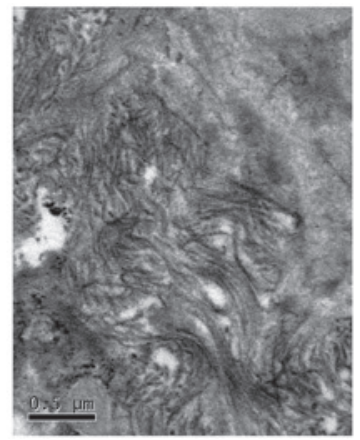

C

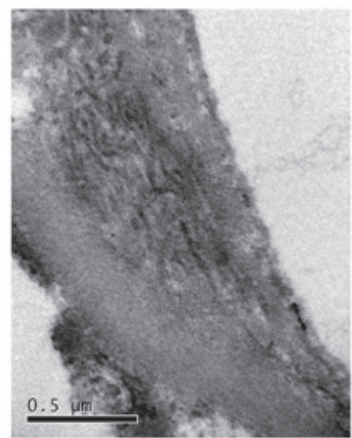

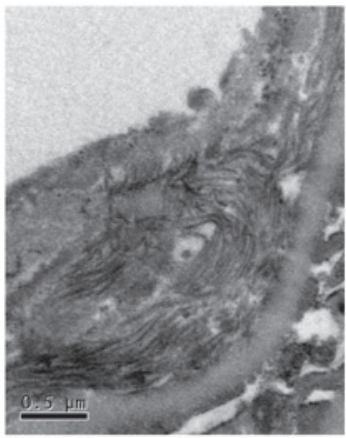

Figure 3. Second renal biopsy. (A) magnification, x10,000 and (B) magnification, x15,000 electron microscopic images revealing collagen fibrils (40-60 nm) deposists in the mesangial matrix. (C) Electron microscopic image revealing collagen fibrils $(40-60 \mathrm{~nm})$ deposists in a loose layer of the basal membrane (magnification, x15,000). (D) Electron microscopic image revealing collagen fibrils $(40-60 \mathrm{~nm}$ ) deposists in the subendothelial space (magnification, $\mathrm{x} 15,000)$.

rescence microscopy and electronic microscopy scanning; however, paraffin sections revealed no glomeruli. Thus, the frozen sections were embedded for light microscopy. From the immunofluorescence microscopy, four glomeruli were observed. $\operatorname{IgM}$ was positive in the mesangium, and $\operatorname{IgA}$, $\mathrm{IgG}, \mathrm{C} 3$ and $\mathrm{Clq}$ were weakly positive in the mesangium. Under light microscopy, the glomeruli exhibited segmentation and mild mesangial hypercellularity. Under electronic microscopy scanning, one glomerulus demonstrated no electron-dense or collagen fibril deposits in the mesangial matrix and subendothelial space (Fig. 1). After being treated with ACE inhibitor for one year, the edema and hypertension improved but the proteinuria and anemia persisted.
The patient was readmitted to the hospital on July 19, 2012. Laboratory results revealed urine protein levels of $1.94 \mathrm{~g} / 24 \mathrm{~h}$ without microscopic hematuria, low hemoglobin levels of $80 \mathrm{~g} / \mathrm{l}$, iron panel consistent with iron deficiency, blood urea nitrogen levels of $7.35 \mathrm{mmol} / \mathrm{l}$, creatinine levels of $94.1 \mu \mathrm{mol} / 1$, uric acid levels of $436.6 \mu \mathrm{mol} / 1$ and C3 levels remained low at $0.468 \mathrm{~g} / \mathrm{l}$. A second renal biopsy was then performed on August 2, 2012. Under light microscopy, there were 24 glomeruli, of which one was completely sclerotic. The remainder presented mild mesangial hypercellularity. The basal membrane was crumpled, and there was fake double-tracks in certain segments. No significant immune complex deposition was identified by Masson staining. Foci 
Table I. General information and clinical manifestations of 20 Chinese cases.

\begin{tabular}{|c|c|c|c|c|c|}
\hline Number & Gender & Age at onset (years) & Family history & First symptom & Blood pressure (mmHg) \\
\hline $1(20)$ & Female & 29 & No & Abnormal urine & $140 / 100$ \\
\hline $2(16)$ & Male & 33 & Yes & Edema & $175 / 130$ \\
\hline $3(16)$ & Male & 34 & Yes & Edema & $190 / 130$ \\
\hline $4(19)$ & Male & 55 & No & Edema & $150 / 85$ \\
\hline $5(17)$ & Female & 29 & No & Edema & $100 / 70$ \\
\hline $6(15)$ & Male & 57 & No & Edema & Normal \\
\hline $7(15)$ & Male & 35 & No & Proteinuria & Normal \\
\hline $8(18)$ & Male & 62 & No & Proteinuria & $160 / 100$ \\
\hline $9(21)$ & Female & 8 & Yes & Edema & $210 / 130$ \\
\hline $10(21)$ & Female & 45 & No & Edema & $140 / 100$ \\
\hline $11(21)$ & Male & 29 & No & Abnormal urine & $170 / 105$ \\
\hline $12(21)$ & Female & 29 & No & Abnormal urine & $140 / 100$ \\
\hline $13(21)$ & Female & 47 & No & Abnormal urine & $130 / 80$ \\
\hline $14(21)$ & Female & 19 & No & Edema & $140 / 100$ \\
\hline $15(21)$ & Male & 39 & No & Abnormal urine & $170 / 100$ \\
\hline $16(21)$ & Female & 59 & No & Edema & $160 / 90$ \\
\hline $17(21)$ & Female & 56 & No & Abnormal urine & $150 / 73$ \\
\hline $18(21)$ & Female & 28 & No & Abnormal urine & $130 / 80$ \\
\hline $19(21)$ & Male & 57 & No & Edema, hypertension & $150 / 90$ \\
\hline $20(21)$ & Male & 40 & No & Hypertension & $200 / 102$ \\
\hline
\end{tabular}

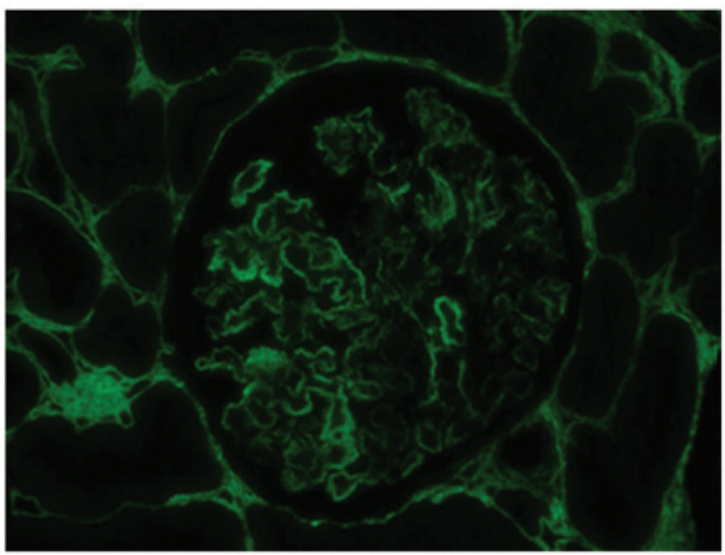

Figure 4. Second renal biopsy: Immunofluorescence using an antibody to type III collagen indicating positivity in numerous capillary walls and mesangial regions.

of interstitial fibrosis and arteriolar hyaline deposits were identified (Fig. 2). By immunofluorescence microscopy, there were four glomeruli. IgA, IgM, IgG and complement C3 were all negative. By electronic microscopy scanning there was one glomerulus, which revealed expansive mesangium, but no proliferative mesangial cells or endothelial cells. Part of the basal membrane demonstrated irregular thickening. There were magnanimous collagen fibrils $(40-60 \mathrm{~nm})$ in the mesangial matrix and a loose layer of the basal membrane and subendothelial space, with no electron-dense deposits (Fig. 3). Immunofluorescence staining of the abnormal extracellular infiltrate for type III collagen was positive (Fig. 4).

\section{Discussion}

In the human kidney, type III collagen, a structural protein of the extracellular matrix, is present only in the interstitium and blood vessels and not in the glomerulus. Accumulation of type III collagen fibrils within the mesangial matrix and subendothelial space is a typical feature of collagen type III glomerulopathy. No more than forty published cases have been reported in the English literature (6); the majority are from Japan (2,7-9), with isolated cases from Canada (10), Italy (3), Slovakia (11) and Brazil $(12,13)$. In this article, we reported the first case in China with two completely different renal biopsies.

We described the case of a 12-year-old male, without a significant family history, who presented with hypertension, anemia, renal insufficiency and hypocomplementemia. Inherited factor $\mathrm{H}$ deficiency is associated with collagen type III glomerulopathy and results in hypocomplementemia (14). However, factor $\mathrm{H}$ was not detected in the present case. In the first renal biopsy, there was only segment and mild mesangial hypercellularity in the glomeruli. No electron-dense or collagen fibrils were identified by electronic microscopic scanning. Notably, in the second renal biopsy, magnanimous collagen fibrils $(40-60 \mathrm{~nm})$ in the mesangial matrix and a loose layer of the basal membrane and subendothelial space were identified and confirmed as type III collagen fibrils by immunofluorescence. In the same patient, the results from two renal biopsies were completely different. We speculate that in the early stage, type III collagen fibril deposits in the glomeruli were focal. In the first renal biopsy, only one tiny fragment with four glomeruli was taken and hence the location where the type III collagen fibrils were deposited was possibly 
Table II. Laboratory results of 20 Chinese cases.

\begin{tabular}{lccccccc}
\hline Number & $\begin{array}{c}\text { Hematuria } \\
(\mathrm{million} / \mathrm{ml})\end{array}$ & $\begin{array}{c}\text { Proteinuria } \\
(\mathrm{g} / 24 \mathrm{~h})\end{array}$ & $\begin{array}{c}\text { Hemoglobin } \\
(\mathrm{g} / \mathrm{l})\end{array}$ & $\begin{array}{c}\text { Serum creatinine } \\
(\mu \mathrm{mol} / \mathrm{l})\end{array}$ & Albumin $(\mathrm{g} / \mathrm{l})$ & $\begin{array}{c}\text { Cholesterol } \\
(\mathrm{mmol} / \mathrm{l})\end{array}$ & $\begin{array}{c}\text { Triglycerides } \\
(\mathrm{mmol} / \mathrm{l})\end{array}$ \\
\hline 1 & 10 & 0.94 & 106 & 71.6 & 37.5 & 5.73 & 2.25 \\
2 & 0 & 2.1 & 117 & 110 & 38 & 6.65 & 4.17 \\
3 & 0 & 6.38 & 44 & 313 & 20 & 3.68 & 0.62 \\
4 & 0 & 24.44 & 134 & 434.3 & 16.8 & 15.1 & 11.83 \\
5 & 0 & 3.03 & 152 & Normal & 12 & 12.31 & 2.09 \\
6 & 0 & 5.6 & 100 & 150 & 23.9 & 4.77 & 2.51 \\
7 & 0 & 6.5 & Normal & Normal & 29 & 4.7 & 1.8 \\
8 & 0 & 2.6 & 142 & 139 & - & - & - \\
9 & 0 & 0.24 & 91 & 76.91 & 39.4 & 5.82 & 1.48 \\
10 & 0 & 0.28 & 129 & 137.91 & 41.4 & 3.13 & 1.01 \\
11 & 0 & 1.19 & 164 & 89.28 & 50.8 & 5.09 & 2.01 \\
12 & 0 & 0.77 & 101 & 71.6 & 37.5 & 5.73 & 2.25 \\
13 & 0 & 1.3 & 118 & 52.16 & 42.7 & 4.38 & 3.68 \\
14 & 10 & 0.42 & 138 & 53.04 & 35.7 & 5.91 & 1.33 \\
15 & 0 & 6 & 118 & 119.34 & 33.7 & 6.86 & 2.23 \\
16 & 0 & 3.27 & 92 & 106.96 & 25.5 & 6.41 & 1.12 \\
17 & 62 & 3.68 & 91 & 74.26 & 34 & 6.07 & 2.42 \\
18 & 19 & 1.65 & 105 & 46.85 & 36.3 & 4.58 & 2.49 \\
19 & 0 & 3.69 & 79 & 174.15 & 27.8 & 7.31 & 2.31 \\
20 & 30 & 2.13 & 90 & 351.83 & 41.9 & 4.88 & 23.8 \\
\hline
\end{tabular}

missed. As the disease progressed, an increasing number of collagen fibrils were deposited in the glomeruli. Thus, we were able to diagnose collagen type III glomerulopathy with the second renal biopsy. Below, we review all cases in China since 1979 (15-21) for an improved understanding of collagen type III glomerulopathy.

In China, the cases of 10 female patients and 10 male patients have been reported (Tables I and II). Three patients had a family history of kidney disease. The youngest patient was eight years old and the oldest was 62 . The average age of onset of the disease was $39.50 \pm 14.83$ years. The early presentation was usually edema and proteinuria. Seventy-five percent of the patients developed hypertension. Thirty percent of the patients had nephrotic syndrome and $20 \%$ had mild hematuria. Approximately $50 \%$ of the patients were anemic, and $45 \%$ had renal insufficiency. There was no correlation between creatinine and hemoglobin or blood pressure. Complement C3 was normal and serum levels of factor $\mathrm{H}$ were not detected in all 20 cases. Notably elevated procollagen type III peptide was detected in only two cases. The kidneys were enlarged in the majority of cases.

A renal biopsy was performed in all 20 cases. Under light microscopy, the volume of the majority of renal glomeruli was increased without substantial hypercellularity. The glomerular lesions could be roughly divided into two types; one with nodular changes without substantial hypercellularity, and the other with no nodular changes. In both types, the glomerular subendothelial space appeared loose and widened. Diffuse fake two-track was observed in the capillary loop. However, in the first type, the subendothelial space was found to be filled with a homogeneous and eosinophilic substance by periodic acid-Schiff staining, and filled with a substance which was dyed green by Masson staining, which caused a narrowing of the capillary lumina. Foci of interstitial fibrosis and tubular atrophy were observed in all cases, while arteriolar hyaline was observed only in cases with hypertension. By immunofluorescence microscopy, in the majority of cases, antibodies (IgA, IgG and $\operatorname{IgM}$ ) and components of the complement $(\mathrm{Clq}$ and C3) were negative. However, IgM or C3 non-specific deposition was observed in seven cases and $\operatorname{Ig}$ A was positive in the mesangial area in four cases. By electronic microscopy scanning, collagen fibrils (diameter 40-100 nm) were identified in the mesangial matrix and subendothelial space and type III collagen fibril was confirmed by immunofluorescence staining. In addition, the fusion of foot processes was also common. In cases with IgA deposition, electron-dense material was identified in the mesangial area. No extra-renal deposition of type III collagen fibrils was identified in any of the 20 cases.

In early studies, prednisone was used for treatment, but with little effect (15). In later studies, the main treatments were angiotensin-converting enzyme inhibitor and restriction of protein intake. The median follow-up time in a study of 12 cases was $20.83 \pm 7.86$ months (6-35 months); new renal insufficiency developed in one patient who had normal creatinine at diagnosis, while slow progression of renal dysfunction was observed in four patients who had abnormal creatinine in the beginning. In one case, malignant hypertension quickly progressed to end-stage renal disease (21).

In our review, the Chinese patients were mostly adults, which contrasts with previous reviews where all the patients 
are extremely young; six patients were younger than two years old in a study by Gubler et al (22). Persistent proteinuria is the most common presentation of collagen type III glomerulopathy. Among those with proteinuria, 30\% of the Chinese patients had nephrotic syndrome, while $60 \%$ of patients fell into the nephritic range in the English literature (16). Hypertension was also prominent (in $75 \%$ of cases), which is similar to the results in the English literature (reported in two-thirds of all cases) (16). Forty-five percent of patients in China presented with elevated creatinine levels, while renal function was usually normal or slightly decreased at presentation in previous studies (6). This may be associated with the late onset of the disease in Chinese patients.

Complement declining due to a deficiency of factor $\mathrm{H}$ is thought to be a manifestation of collagen type III glomerulopathy (14), but we noted that this was rare in Chinese cases. In English publications, cases have occasionally been described within families $(8,22,23)$; hence, it has been assumed that collagen type III glomerulopathy may have an autosomal recessive trait (24). In Chinese literature, cases within the same family were also noted (16). However, the pathogenesis of collagen type III glomerulopathy remains unclear. Some consider it to be a systemic disease $(9,24)$, while others consider it a primary renal disease $(2,3)$. In China, procollagen type III peptide increased notably in the cases reported by Chen et al (16) and cases with coronary heart disease, thyroid tumor, gallbladder polyps or multiple swollen lymph nodes in the mediastinum have been reported (21); however, extra-renal type III collagen fibril deposition was not identified in any of the cases reported. The pathological manifestation of collagen type III glomerulopathy is significant (25). The pathology of collagen type III glomerulopathy in Chinese cases is similar to that observed in other ethnicities, with the exception that, in China, certain cases were revealed to be IgA-positive under immunofluorescence microscopy, and electron-dense material was identified in the mesangial area. This may be due to the fact that IgA nephropathy is common in China. It is not hard to establish a definite diagnosis of collagen type III glomerulopathy with typical immunohistochemical staining for specific collagen types; however, there is no effective treatment available.

In conclusion, collagen type III glomerulopathy is a rare glomerular disease, with 21 cases reported in China to date. The onset age, clinical manifestation and pathological features of the disease are not exactly the same worldwide.

\section{References}

1. Arakawa M, Hueki H, Sato M, Yamashita K and Nakashima S: Idiopathic mesangiodegenerative glomerulonephropathy: A proposal of a new glomerular disease. Jpn J Nephrol 21: 1404, 1979.
2. Ikeda K, Yokoyama H, Tomosugi N, Kida H, Ooshima A and Kobayashi K: Primary glomerular fibrosis: A new nephropathy caused by diffuse intraglomerular increase in atypical type III collagen fibers. Clin Nephrol 33: 155-159, 1990.

3. Imbasciati E, Gherardi G, Morozumi K, et al: Collagen type III glomerulopathy: a new idiopathic glomerular disease. Am J Nephrol 11: 422-429, 1991.

4. Gibson W and More AR: Glomerular pathology: recent advances. J Pathol 184: 123-129, 1998.

5. Proesmans W, Van Dyck M and Devriendt K: Nail-patella syndrome, infantile nephrotic syndrome: complete remission with antiproteinuric treatment. Nephrol Dial Transplant 24: 1335-1338, 2009.

6. Alchi B, Nishi S and Narita I: Collagenofibrotic glomerulopathy: clinicopathologic over-view of a rare glomerular disease. Am J Kidney Dis 49: 499-506, 2007.

7. Yoshioka K, Takemura T, Tohda M, et al: Glomerular localization of type III collagen in human kidney disease. Kidney Int 35: 1203-1211, 1989

8. Tamura H, Matsuda A, Kidoguchi N, Matsumura O, Mitarai T and Isoda K: A family with two sisters with collagenofibrotic glomerulonephropathy. Am J Kidney Dis 27: 588-595, 1996.

9. Yasuda T, Imai H, Nakamoto Y, et al: Collagenofibrotic glomerulopathy: a systemic disease. Am J Kidney Dis 33: 123-127, 1999.

10. Dombros N and Katz A: Nail-patella like renal lesion in the absence of skeletal abnormalities. Am J Kidney Dis 1: 237-240, 1982.

11. Bernasovská G, Demes M, Oksa A, et al: Collagenfibrotic glomerulopathy - rare glomerulonephritis. Vnitr Lek 52: 1200-1204, 2006 (In Czech).

12. Bichuette-Custodio F, Castro E, Teixeira V and Reis MA: Collagenofibrotic glomerulopathy: A description of two cases. Abstr book World Congress Nephrol 327, 2007.

13. Ferreira RD, Custódio FB, Guimarães CS, Corrêa RR and Reis MA: Collagenofibrotic glomerulopathy: three case reports in Brazil. Diagn Pathol 4: 33, 2009.

14. Vogt BA, Wyatt RJ, Burke BA, Simonton SC and Kashtan CE: Inherited factor $\mathrm{H}$ deficiency and collagen type III glomerulopathy. Pediatr Nephrol 9: 11-15, 1995.

15. Zhou FD, Zhou WZ, Huang CX, et al: Collagen type III glomerulopathy with two case reports. Chin J Nephrol 14: 75-79, 1998.

16. Chen N, Xu YW, Pan XX, et al: A family with two brothers with Collagen type III glomerulopathy. Chin J Nephrol 21: 645-648, 2005.

17. Liu XG, Zhao ZH, Liu Y, Zhang ZG and Guo MY: Collagen type III glomerulopathy: A case report. Chin J Nephrol 21: 336-337, 2005.

18. Lu DQ: A case report of Collagen type III glomerulopathy. Chin J Integr Tradit and Western Nephrol 6: 113, 2005.

19. Gu FF and Shang HP: Collagen type III glomerulopathy: A case report. Med J Liaoning 21: 45-46, 2007.

20. Pu QD and Kuang SQ: Collagen type III glomerulopathy: A case report. China Foreign Med Treat 23: 57-58, 2010.

21. Chen HP, Xu F and Huang Q: Collagen type III glomerulopathy: clinical and pathological features. J Nephrol Dialy Transplant 20: 522-529, 2011.

22. Gubler MC, Dommergues JP, Foulard M, et al: Collagen type III glomerulopathy: a new type of hereditary nephropathy. Pediatr Nephrol 7: 354-360, 1993.

23. Salcedo JR: An autosomal recessive disorder with glomerular basement membrane abnormalities similar to those seen in the nail patella syndrome: report of a kindred. Am J Med Genet 19: 579-584, 1984.

24. Soni SS, Gowrishankar S, Nagarik AP, et al: Collagenofibrotic glomerulopathy in association with Hodgkin's lymphoma. Saudi J Kidney Dis Transpl 22: 126-129, 2011.

25. Cohen AH: Collagen type III glomerulopathies. Adv Chronic Kidney Dis 19: 101-106, 2012. 